\title{
Evaluación de la rentabilidad y competitividad de los sistemas de producción de ovinos en la región de Libres, Puebla
}

\section{Evaluation of the profitability and competitiveness of sheep production systems in the region of Libres, Puebla}

Carla Cristina Díaz-Sánchez ${ }^{\mathrm{a}}$

José Luis Jaramillo-Villanueva ${ }^{\mathrm{b}}$

Ángel Bustamante-González

Samuel Vargas-López ${ }^{b^{*}}$

Adriana Delgado-Alvarado ${ }^{\mathrm{b}}$

Omar Hernández-Mendo ${ }^{c}$

Miguel Ángel Casiano-Ventura ${ }^{b}$

${ }^{a}$ Estudiante de maestría del Posgrado en Estrategias para el Desarrollo Agrícola Regional del Colegio de Postgraduados, Campus Puebla, Puebla. México.

${ }^{\mathrm{b}}$ Colegio de Postgraduados, Campus Puebla, Puebla. México.

${ }^{\mathrm{c}}$ Colegio de Postgraduados, Campus Montecillo, Estado de México. México.

* Autor de correspondencia: svargas@ colpos.mx

\section{- Resumen:}

Con el objetivo de evaluar el grado en que los sistemas de producción de ovinos son competitivos y presentan ventajas comparativas, se entrevistaron a 139 productores, y se dio seguimiento a seis rebaños de tres sistemas de producción durante siete meses, en la región de Libres, Puebla. Se registró información de costos, infraestructura, actividad agrícola, 
mano de obra, producción y venta de ovinos. Para determinar la rentabilidad y la competitividad se utilizó la Matriz de Análisis de Política. El sistema de producción con alimentación en corral fue el más rentable y competitivo de acuerdo con los indicadores de rentabilidad privada, Relación del Costo Privado, Coeficiente de Rentabilidad Privada y la Relación del Costo-Beneficio Privado. El costo de los factores de producción fue mayor en el sistema de producción de corderos en pastoreo y fue el de menor competitividad. La Relación Costo-Beneficio Social indicó ineficiencia para reemplazar las importaciones. La producción de ovinos tuvo una estructura de mercado oligopsónica, producto de las deficiencias institucionales y ausencia de políticas de protección. Los productores utilizaron como criterio de venta el peso adulto, aunque la venta de los corderos inició entre los 30 y 90 días. Es recomendable realizar el análisis de la función de producción, para determinar la viabilidad de la venta de cordero destetado, como una opción para aumentar las ganancias de los productores y la competitividad de estos sistemas.

- Palabras clave: Análisis de costos, Ingresos, Política, Producción de cordero.

\begin{abstract}
- Abstract:
With the objective to evaluate the degree of competitiveness and comparative advantage of local lamb production systems, a sample of 139 sheep producers were interviewed, and six herds of three production systems were under observation for 7 mo in the Libres region of Puebla, Mexico. Information was recorded on costs, infrastructure, agricultural activity, labor, production and sale of sheep. The profitability and competitiveness was determined with the Policy Analysis Matrix. The system of production on feeding indoors was the most profitable and competitive according to the Index of Private Profitability, Private Cost Ratio, Private Profitability Ratio and Private Cost-Benefit Ratio. The cost of the factors of production was higher in the lamb grazing system, being of the less competitive. The Social Cost-Benefit Ratio (CSBR) indicated inefficiency of systems to replace imports. The production of sheep had an oligopsonic market structure due to institutional deficiencies and absence of protection policies oriented to this sector. The producers used as the criterion of sale the mature weight, although the sale of the lambs began between 30 and $90 \mathrm{~d}$ old. It is advisable to perform an analysis of the production function, to determine the viability of the sale of weaned lambs as an option to increase the profits of the producers and the competitiveness of these systems.
\end{abstract}

- Key words: Cost analysis, Income, Policies, Lamb production.

Recibido 22/05/2017.

Aceptado 14/10/2017. 


\section{VIntroducción}

En México la producción de ovinos es una alternativa económica para enfrentar la pobreza en el medio rural, debido a la cantidad de ingreso generado y al número de productores involucrados en esta actividad ${ }^{(1)}$. El inventario nacional es de 8.7 millones de ovinos en 50,000 unidades de producción y de éstas, $34 \%$ de los productores viven de la especie ${ }^{(2)}$. La zona templada del centro del país tiene una amplia tradición en la producción de ovinos y demanda $85 \%$ de la carne de cordero consumida a nivel nacional ${ }^{(3)}$. En esta zona, el estado de Puebla produce 636,379 ovinos, en 2,515 unidades de producción ${ }^{(4)}$, de los distritos de Zacatlán, Libres y Teziutlán, donde se produce $72 \%$ de las 4,125 t de carne de cordero ${ }^{(5)}$.

En el país, los sistemas pastoriles tradicionales son los que abastecen de carne de ovino a los mercados locales y presentan diferente nivel tecnológico, capacidad productiva y uso de recursos $^{(6)}$. En estos sistemas, los ovinos representan una alternativa rentable por el buen precio de venta del cordero y la alta demanda ${ }^{(7)}$, la cual se cubre con importaciones ${ }^{(8)}$. Los estudios económicos de la producción de ovinos en el centro del país registraron ganancias para el productor de $40 \%$ por kilo de carne $(\$ 27.3)$, respecto al precio final al consumidor, mientras que el intermediario obtuvo $60 \%(\$ 38.7)^{(9)}$; y el costo de producción de $1 \mathrm{~kg}$ de carne fue de $\$ 32.5^{(10)}$. El canal de comercialización más común fue de productor a acopiador, barbacoyero y consumidor final, con una relación beneficio-costo para el productor de 1.05 y para el barbacoyero de $5.64^{(9)}$.

Los sistemas de producción de ovinos tradicionales compiten con grandes productores internacionales, debido a la política macroeconómica de apertura comercial vigente en México, y con la desventaja de estar produciendo en un contexto de políticas nacionales orientadas a la descentralización de las actividades de desarrollo, y al impulso de aquellos productores rurales con potencial competitivo; lo cual determina que el mercado y las políticas tengan impacto sobre la competitividad de este sector ${ }^{(11)}$.

La competitividad se define como la capacidad de un país, región, industria o empresa, de generar ganancias y mantenerse en el mercado. Tiene como sustento a nivel microeconómico, la productividad y la eficiencia. A nivel macroeconómico, está determinada por la tasa de interés, la tasa de cambio, la balanza comercial y la política económica ${ }^{(12,13)}$.

Existen diferentes metodologías con sustento en la teoría económica para medir la competitividad, como son la participación en el mercado, ventaja relativa de las exportaciones, competitividad revelada, el enfoque evolucionista basado en la productividad 
y la distribución del ingreso, y la Matriz de Análisis de Política (MAP) ${ }^{(13,14)}$. La MAP a diferencia de las otras metodologías, mide la eficiencia de la producción, la ventaja comparativa y los impactos de la política económica sobre el sistema de producción ${ }^{(15)}$; genera resultados comprensibles para los tomadores de decisiones y tiene consistencia teórica. El método permite medir los impactos de las políticas públicas actuales, y de los cambios de precio sobre la ganancia de los productores a nivel individual y a nivel de una industria, así como las transferencias entre agentes económicos. También, permite calcular la rentabilidad para los propietarios de las inversiones y para la sociedad. Es decir, la MAP tiene dos identidades fundamentales: la primera, determina el nivel de ganancia como la diferencia entre los ingresos y los costos de producción; la segunda, mide los efectos de política gubernamental y de las distorsiones de mercado.

Es muy importante determinar la competitividad a nivel industria y sistema productivo para identificar el tipo de productores, clasificados por las tecnologías que usan, los cultivos o especies que explotan y las zonas agroclimáticas en que se ubican, y si son competitivos bajo las políticas actuales, lo que permite una toma de decisiones informada ${ }^{(16,17)}$.

El objetivo del estudio fue evaluar el grado en que los sistemas de producción de ovinos en la región de Libres, Puebla, México, son competitivos y presentan ventajas comparativas, permitiendo así determinar la rentabilidad privada, económica, los efectos de política y las distorsiones del mercado o divergencias.

La hipótesis que guio el presente estudio, fue que los sistemas locales de producción de ovinos presentan diferencias en su competitividad y ventaja comparativa, debido al uso de factores internos.

\section{Material y métodos}

\section{- Área de estudio}

El estudio se realizó en la región de Libres, Puebla, México, a $19^{\circ} 21^{\prime}$ y $19^{\circ} 33^{\prime} \mathrm{N}$ y $97^{\circ} 32^{\prime}$ y $97^{\circ} 48^{\prime} \mathrm{O}$. La altitud osciló entre 2,320 y 3,400 msnm. El clima es templado subhúmedo con lluvias en verano, semifrío subhúmedo con lluvias en verano y semiseco templado. La temperatura media anual es de $18{ }^{\circ} \mathrm{C}$ y la precipitación pluvial de 400 a $900 \mathrm{~mm}$ anuales ${ }^{(18)}$. 


\section{- Registro de información}

El registro de información se realizó en dos momentos. En el primero, mediante muestreo simple aleatorio, empleando la fórmula propuesta por Scheaffer et $a l^{(19)}$, con margen de error del $8.3 \%$ y máxima varianza para preguntas dicotómicas $(0.25)$; se seleccionó una muestra de 139 productores de ovinos para registrar los activos productivos, manejo, costos de producción, ventas, actividad agrícola y mano de obra. Con esta información se identificaron tres sistemas de producción: sólo pastoreo de agostaderos y rastrojos (S1), pastoreo de agostaderos y praderas (S2) y alimentación en corral (S3).

En el segundo momento, se dio seguimiento, de noviembre de 2015 a junio de 2016, a seis unidades de producción, dos para cada sistema, en la comunidad de Nuevo México del municipio de Libres, Puebla, que fue donde se encontraron los tres sistemas de producción previamente identificados. En la selección de las unidades de producción para el seguimiento se consideró la disponibilidad del productor para acompañar al técnico en el registro de información, y que el tamaño del rebaño fuera similar al que previamente se determinó con la primera base de datos para la región. Las características de las unidades de producción en cada sistema se describen a continuación:

S1. Sólo pastoreo de agostaderos y rastrojos. Rebaño promedio de 83 ovejas de cría. Los ovinos se alimentaron de la vegetación natural y rastrojos de los cultivos del maíz. En la vegetación natural de las áreas de agostadero estuvieron presentes las especies Medicago lupulina L. (alfalfilla), Erodium cicutarium L'Herit (alfilerillo); Taraxacum officinale (diente de león); Sonchus oleraceus (lechuguilla) y Brassica campestris L. (nabo silvestre). El pastoreo duró $8 \mathrm{~h}$ en promedio, 0700 a $1500 \mathrm{~h}$.

S2. Sistema de pastoreo en agostaderos y praderas. Rebaño promedio de 125 ovejas de cría. $\mathrm{El}$ agostadero fue similar al utilizado en el S1. Las especies cultivadas en las praderas fueron Dactylis glomerata (pasto ovillo), Brassica campestris L. (nabo silvestre), Triticum aestivum (trigo) y Medicago sativa (alfalfa). El pastoreo se realizó de 0900 a $1300 \mathrm{~h}$ en las praderas y de las 1300 a $1500 \mathrm{~h}$ en el agostadero.

S3. Sistema de alimentación con rastrojo, alfalfa y grano de maíz en corral. Rebaño promedio de 107 ovejas de cría. Los corderos después del destete (60 días) fueron estabulados y alimentados con $60 \%$ de rastrojo y alfalfa y $40 \%$ de grano de maíz molido. 
En una ficha se registró quincenalmente en cada rebaño la información del uso de granos, sales minerales, medicinas y vacunas, valor de la renta de la tierra, mano de obra para el pastoreo y el manejo de los ovinos, similar a otros estudios ${ }^{(20)}$. Adicionalmente, se registró la depreciación del pie de cría e instalaciones.

\section{- Análisis de datos}

La MAP se integró de acuerdo a la metodología de Salcedo ${ }^{(21)}$. Se calcularon los costos de los insumos comercializables y los factores de producción a partir de la información recabada en campo, como fue realizado en otros estudios ${ }^{(17)}$. Los conceptos de ingreso y costos fueron los siguientes:

Insumos comercializables. Son los que se comercializan en las condiciones actuales de apertura comercial y tienen una cotización internacional, que para este estudio fueron los alimentos, medicamentos y el cordero. Para el presupuesto a precios sociales se utilizaron las bases de datos del Sistema Nacional de Información de Integración de Mercados ${ }^{(22)}$.

Factores internos. Son los que intervienen en la producción, pero que no son comercializados internacionalmente y, por ello, su precio se determinó en el mercado interno por la interacción de oferta y demanda, como fue la mano de obra, renta de la tierra, depreciación de instalaciones y depreciación del valor del pie de cría. El costo por hora de trabajo para el manejo de los ovinos fue de $\$ 15.00$ y se consideró el mismo valor a precio privado y social, debido a que los productores no tienen alternativas de empleo en la región con un mejor salario. El costo del factor tierra fue por el pago de la renta de agostaderos ${ }^{(23)}$. El factor capital incluyó la depreciación de las instalaciones y la depreciación del valor del pie de cría. El costo de otros productos y servicios, como luz y agua, no se consideraron, al no contar la unidad de producción con un contrato exclusivo para los ovinos ${ }^{(17)}$. El precio de venta regional del cordero adulto utilizado para el cálculo de los ingresos de la MAP fue de \$43.0, $\$ 42.0$ y $\$ 44.0$ para S1, S2 y S3, respectivamente. Con el promedio del ingreso, costo de los insumos y de los factores de la producción de las unidades de producción se integró la MAP para los tres sistemas, de acuerdo a la estructura del Cuadro $1^{(17,21)}$. 
Cuadro 1: Estructura y estimación de parámetros de la Matriz de Análisis de Política

\begin{tabular}{lcccc}
\hline Concepto & $\begin{array}{c}\text { Bienes } \\
\text { comercializables }\end{array}$ & Factores de producción & Rentabilidad \\
\hline $\begin{array}{l}\text { Ingreso por venta } \\
\text { de productos }\end{array}$ & $\begin{array}{c}\text { Costos de } \\
\text { insumos }\end{array}$ & $\begin{array}{c}\text { Costo de mano de obra, } \\
\text { tierra y capital }\end{array}$ & \\
Presupuesto privado & A & B & C & D= A-B-C \\
Presupuesto social & E & F & G & H= E-F-G \\
\hline Divergencias & I=A-E & J=B-F & K=C-G & L=D-H \\
\hline I= divergencias a través de productos; J= divergencias a través de insumos; $K=$ divergencias a través de los factores de \\
producción; $L=$ divergencias netas. Modificado de Monke y Pearson (17,18).
\end{tabular}

A partir del presupuesto a precios privados de la MAP se calcularon los siguientes indicadores de rentabilidad: ingreso bruto $(\$ / \mathrm{kg})$, costo total $(\$ / \mathrm{kg})$, ganancia neta $(\$ / \mathrm{kg})$ y el Coeficiente de Rentabilidad Privada $(\mathrm{CRP}=\mathrm{D} /(\mathrm{B}+\mathrm{C})$. Con el $\mathrm{CRP}$ se evaluaron las ganancias por cada peso invertido ${ }^{(24)}$. Con el presupuesto a precios sociales se calculó la Relación Costo-Beneficio Social $\left(\mathrm{RCBS}=\mathrm{E} /(\mathrm{F}+\mathrm{G})\right.$ para comparar la eficiencia entre los sistemas ${ }^{(17)}$.

Los indicadores de competitividad estimados fueron la Relación Costo-Beneficio Privado $\left(\mathrm{RCBP}=\mathrm{A} /(\mathrm{B}+\mathrm{C})\right.$ y la Relación del Costo Privado $\left(\mathrm{RCP}=\mathrm{C} /(\mathrm{A}-\mathrm{B})^{(16)}\right.$. La RCBP comparó la competitividad de los sistemas a precios privados ${ }^{(17)}$. Con la RCP se midió la competitividad de los sistemas que generan el mismo producto, y determinó el límite donde se cubrió el pago de los factores internos ${ }^{(24,25)}$.

Adicionalmente, para comparar el efecto de las políticas entre los sistemas se utilizó el Coeficiente de Protección Nominal del Producto $(\mathrm{CPNP}=\mathrm{A} / \mathrm{E})$ y el Coeficiente de Protección Nominal de Insumos (CPNI= B/F), los cuales explicaron la diferencia de los precios privados con los precios sociales; el Coeficiente de Protección Efectiva $(\mathrm{CPE}=(\mathrm{A}-\mathrm{B}) /(\mathrm{E}-\mathrm{F})$ que midió el efecto conjunto de las divergencias de las políticas sobre los productos comercializables y no comercializables; y el Coeficiente de Rentabilidad $(C R=D / H)$ que mide el impacto de todas las divergencias sobre los ingresos ${ }^{(16)}$. Una divergencia hace que el precio real del mercado o privado difiera de un precio de eficiencia o social, el cual es resultado de políticas distorsionantes o fallas de mercado en los sistemas productivos ${ }^{(26)}$; así mismo, muestra la ineficiencia en el uso de los recursos ${ }^{(27)}$. 


\section{Resultados y discusión}

\section{- Rentabilidad y competitividad •}

En la región de Libres, Puebla, el valor de los insumos comercializables representó $27.7 \%$ para S3, $18.6 \%$ para S2 y $0.5 \%$ para S1 (Cuadro 2). Por el contrario, el costo de los factores de producción por concepto de la mano de obra fue mayor en S1 (70.4\%) y el costo de renta de la tierra fue mayor en S2 (32.6\%), pero el costo del capital fue más alto en S3 (4.7 \%).

Cuadro 2: Costos de producción promedio en sistemas locales de ovinos de la región de Libres, Puebla

\begin{tabular}{lcccccc}
\hline & \multicolumn{5}{c}{ Sistema de producción } \\
\cline { 2 - 7 } Concepto & \multicolumn{2}{c}{ S1 } & \multicolumn{2}{c}{ S2 } & \multicolumn{2}{c}{ S3 } \\
\cline { 2 - 7 } Insumos comercializables & 285.1 & 0.5 & $13,070.9$ & 18.6 & $12,165.5$ & 27.7 \\
Mano de obra (trabajo) & $43,800.0$ & 70.4 & $32,850.0$ & 46.7 & $17,100.0$ & 38.9 \\
Tierra & $17,506.8$ & 28.1 & $22,982.4$ & 32.6 & $12,604.2$ & 28.7 \\
Capital (inversión/depreciación) & 601.7 & 1.0 & $1,448.3$ & 2.1 & $2,062.0$ & 4.7 \\
Costo total de producción & $62,193.6$ & & $70,351.6$ & & $43,931.7$ & \\
\hline
\end{tabular}

S1= pastoreo de agostaderos y rastrojos; S2= pastoreo de agostaderos y praderas; S3= alimentación en corral.

En el Cuadro 3 se presenta la estructura de la MAP. Los ingresos y costos fueron diferentes para los tres sistemas de producción de ovinos. La rentabilidad privada fue positiva para los tres sistemas, explicada por los bajos costos de insumos y factores de la producción utilizados con relación a los ingresos por la venta de ovinos ${ }^{(17,28)}$. El producto vendido fue el cordero finalizado, aunque la venta inició de 30 a 90 días después del nacimiento, lo cual representa una oportunidad para realizar un análisis de la función de producción del cordero al destete, y así reducir la carga ganadera y los costos privados en la unidad de producción. La rentabilidad social fue mayor a la rentabilidad privada en los tres sistemas, explicada por el mayor precio del cordero en el mercado de importación. 
Cuadro 3: Matriz de Análisis de Política de sistemas de producción de ovinos de la región de Libres, Puebla

\begin{tabular}{lcccc}
\hline Concepto & $\begin{array}{c}\text { Bienes } \\
\text { comercializables }\end{array}$ & \multicolumn{2}{c}{ Factores de producción } & Rentabilidad \\
\hline & $\begin{array}{c}\text { Ingreso por venta } \\
\text { de productos }(\$)\end{array}$ & $\begin{array}{c}\text { Costos de } \\
\text { insumos }(\$)\end{array}$ & $\begin{array}{c}\text { Costo del trabajo, } \\
\text { tierra y capital }(\$)\end{array}$ & \\
\hline S1 & & & & \\
Presupuesto privado & $102,431.2$ & 285.1 & $61,908.5$ & $40,237.7$ \\
Presupuesto social & $113,034.8$ & 285.1 & $58,801.7$ & $53,948.0$ \\
Divergencias & $-10,603.5$ & 0.0 & $3,106.80$ & $-13,710.3$ \\
S2 & & & & \\
Presupuesto privado & $126,307.1$ & $13,070.9$ & $57,280.7$ & $55,955.5$ \\
Presupuesto social & $142,539.9$ & $14,121.4$ & $48,698.3$ & $-23,764.7$ \\
Divergencias & $-16,232.8$ & $-1,050.6$ & $8,582.4$ & \\
S3 & & & & $89,210.7$ \\
Presupuesto privado & $133,142.4$ & $12,165.5$ & $31,766.2$ & $100,123.9$ \\
Presupuesto social & $144,067.6$ & $16,381.6$ & $27,562.0$ & $-10,913.3$ \\
Divergencias & $-10,925.2$ & $-4,216.1$ & $4,204.2$ & \\
\hline
\end{tabular}

$\mathrm{S} 1=$ pastoreo de agostaderos y rastrojos; $\mathrm{S} 2=$ pastoreo de agostaderos y praderas; $\mathrm{S} 3=$ alimentación en corral.

Las divergencias a través del producto (I) fueron negativas para los tres sistemas, y las divergencias a través de los insumos (J) fueron negativas sólo para el S2 y S3. Esto indicó pérdidas económicas para los productores, mencionadas como transferencias de recursos fuera del sistema ${ }^{(16)}$. Las divergencias a través de los factores de producción $(\mathrm{K})$ resultaron en valores positivos para todos los sistemas, principalmente porque el pago de la renta de tierra depende de la cantidad de pastos o rastrojo para alimentar al rebaño. Así mismo, el costo de mano de obra en la región es alto, si se compara con el salario mínimo que rige en México, aspecto común en las actividades agropecuarias del medio rural ${ }^{(16)}$. Además, la eliminación de las barreras comerciales en México de los últimos años no resultó en un aumento de los salarios para trabajadores no calificados, como se esperaba ${ }^{(29)}$.

Las divergencias netas (L) tuvieron valores negativos para los tres sistemas y se explicaron por la presencia de fallas del mercado ${ }^{(27)}$, oligopsonio en este caso, al ser el intermediario quien fijó el precio del cordero a la venta. Esto es común en la comercialización de ovinos en otras regiones de México ${ }^{(7,28)}$. La participación directa en el mercado se afectó por la falta de información de la producción y del precio de los ovinos, lo que coincide con otros estudios de competitividad ${ }^{(30)}$. 
Los indicadores de rentabilidad y competitividad para los tres sistemas se presentan en el Cuadro 4. En los indicadores de rentabilidad, el ingreso bruto por kilo de cordero tuvo un rango de $\$ 41.9$ a $\$ 43.1$, el cual fue el precio final fijado por el intermediario. El costo de producción de un kilo de cordero fue mayor en el S1 (\$26.2) y el menos eficiente, debido al alto costo de mano de obra y renta de tierra. El menor costo por kilo de cordero fue en el S3 (\$14.1), con la mayor ganancia neta (\$28.6) por kilo de cordero producido y se explicó por el menor uso de mano de obra para el manejo de los ovinos.

Cuadro 4: Indicadores de rentabilidad y competitividad de los sistemas de producción de ovinos de la región de Libres, Puebla

\begin{tabular}{lccc}
\hline Indicadores & \multicolumn{3}{c}{ Sistema de producción } \\
\hline & S1 & S2 & S3 \\
\cline { 2 - 4 } Ingreso bruto, $\$ / \mathrm{kg}$ & 43.1 & 41.9 & 42.7 \\
Costo total, \$/kg & 26.2 & 23.3 & 14.1 \\
Ganancia neta, \$/kg & 16.9 & 18.6 & 28.6 \\
Coeficiente de Rentabilidad Privada (CRP) & 0.6 & 0.8 & 2.0 \\
Relación Costo-Beneficio Privado (RCBP) & 1.6 & 1.8 & 3.0 \\
Relación Costo-Beneficio Social (RCBS) & 1.9 & 2.3 & 3.3 \\
Relación del Costo Privado (RCP) & 0.6 & 0.5 & 0.3 \\
\hline
\end{tabular}

$\mathrm{S} 1=$ pastoreo de agostaderos y rastrojos; $\mathrm{S} 2=$ pastoreo de agostaderos y praderas; $\mathrm{S} 3=$ alimentación en corral.

Por el CRP, el S3 (2.0) fue más rentable y el S1 fue el que obtuvo el menor ingreso por peso invertido; similares resultados fueron encontrados en ganado bovino ${ }^{(24)}$. La RCBS fue mayor a la unidad en los tres sistemas, porque el costo de los insumos y de los factores de producción a precio social fueron menores que el ingreso ${ }^{(31)}$. De acuerdo a este indicador, el S1 fue el que utilizó mínimos insumos, comparado con el S2 y S3. Estos resultados de la RCBS explicaron que los sistemas de ovinos en la región de estudio requieren de un porcentaje del producto importado para satisfacer la demanda, lo cual coincide con otros estudios de competitividad ${ }^{(31,32)}$. De acuerdo a la MAP, esto sugiere que a nivel nacional hay desinterés por el desarrollo de la ovinocultura, como se ha mencionado para otras regiones ${ }^{(17)}$. Lo anterior, también puede ser producto de las políticas de México que sólo afectan a los precios internos de los insumos comercializables, a diferencia de las políticas de protección de los países industrializados ${ }^{(33)}$.

Respecto a los indicadores de competitividad, por el valor de la RCBP se determinó que el S2 (1.8) y S3 (3.0) fueron los más rentables y competitivos. El S1 tuvo la menor RCBP (1.6) y similares valores de este indicador se clasificaron como menos rentables ${ }^{(24)}$. En este 
sistema, aunque se cubrió el pago de los factores y se obtuvieron ganancias, el pago por concepto de la mano de obra y la renta de tierra fue alto. Sin embargo, la rentabilidad de los tres sistemas fue mayor a la registrada en sistemas intensivos de ovinos ${ }^{(34)}$. En tanto, por el valor de la RCP el S3 (0.3) fue el más competitivo, por cubrir los costos de los factores de la producción y generar ganancias ${ }^{(25)}$. Esto coincide con valores de la RCP de 0.2 para ovinos en pastoreo y difiere con una RCP de -1.7 para uso de alimento comercial ${ }^{(23)}$.

\section{- Efectos de políticas en la competitividad}

El efecto de políticas ${ }^{(35)}$ en los sistemas de ovinos en la región se determinó con los Coeficientes de Protección, los cuales resultaron en valores menores o iguales a la unidad (Cuadro 5). Los valores en el CPNP menor a la unidad para los tres sistemas fueron resultado del bajo precio interno de los productos, comparado con el precio mundial de importación, lo que se asocia a un tipo de cambio subvaluado derivado de políticas macroeconómicas ${ }^{(16,21)}$. En tanto, los valores del CPNI indicaron que se requiere de subvención para estos sistemas, como fue señalado en otro estudio ${ }^{(17)}$. El valor de este indicador se puede reducir con el otorgamiento de subsidios para la compra de alimento del rebaño, y con esto, se reduciría el pago de este insumo por parte de los productores. El CPE indicó que las políticas de desprotección afectaron a los tres sistemas, mientras que el CR fue bajo debido a las

diferencias entre precios privados y $\operatorname{sociales}^{(17)}$, y específicamente, debido a la no competitividad a precios sociales de los tres sistemas estudiados. 
Cuadro 5: Coeficientes de protección de los sistemas de producción de ovinos de la región de Libres, Puebla

\begin{tabular}{lccc}
\hline & \multicolumn{3}{c}{ Sistema de producción } \\
\hline & S1 & S2 & S3 \\
\cline { 2 - 4 } Coeficiente de Protección Nominal del Producto (CPNP) & 0.9 & 0.9 & 0.9 \\
Coeficiente de Protección Nominal de Insumos (CPNI) & 1.0 & 0.9 & 0.7 \\
Coeficiente de Protección Efectiva (CPE) & 0.9 & 0.9 & 0.9 \\
Coeficiente de Rentabilidad (CR) & 0.7 & 0.7 & 0.9 \\
\hline
\end{tabular}

$\mathrm{S} 1=$ pastoreo de agostaderos y rastrojos; $\mathrm{S} 2=$ pastoreo de agostaderos y praderas; $\mathrm{S} 3=$ alimentación en corral.

\section{\| Conclusiones e implicaciones}

En la región de Libres, Puebla, los sistemas de producción de ovinos fueron rentables, al pagar los factores de producción y obtener ganancias para el productor. La RCP indicó que el sistema con alimentación en corral fue el más competitivo y tuvo ganancias por peso invertido. Los sistemas en pastoreo y pastoreo más pradera fueron menos rentables y competitivos de acuerdo con el CRP y la RCBP, por los altos costos en mano de obra y renta de tierra. El RCBS explicó la incapacidad de los sistemas, con un mayor uso de insumos, para reemplazar las importaciones, lo cual se traduce en pérdidas para el país. El valor de las divergencias y los coeficientes de protección sugieren que la competitividad de los sistemas de la región son afectados por la ausencia de apoyos a la producción, que favorezcan a los productores, tales como políticas que fomenten la productividad vía innovación y capacitación a los productores, y que regulen la acción de los agentes del mercado de ovinos, principalmente a intermediarios. La competitividad de la producción de ovinos en la región de Libres, Puebla, estuvo relacionada con la diferencia en la disponibilidad y en el costo de oportunidad de la mano obra, tierra e insumos.

\section{- Literatura citada}

1. Martínez G, Muñoz R, García M, Santoyo C, Altamirano C, Romero M. El fomento de la ovinocultura familiar en México mediante subsidios en activos: lecciones aprendidas. Agron Mesoam 2011;22(2):367-377. 
2. Medrano J. Recursos animales locales del centro de México. Arch Zootec 2000;49(187):385-390.

3. Rodríguez-Licea G, García-Salazar J, Hernández-Martínez J. Identificación de conglomerados para impulsar las cadenas productivas de carne en México. Agron Mesoam 2016;27(2):353-365.

4. INEGI. Instituto Nacional de Estadística, Geografía e Informática. Censo Agropecuario 2007.

http://www.inegi.org.mx/est/contenidos/proyectos/Agro/ca2007/Resultados_Ejidal/def ault.aspx. Consultado 23 Nov, 2016.

5. SAGARPA. Secretaría de Agricultura, Ganadería, Desarrollo Rural, Pesca y Alimentación. Estudio estratégico: evaluación de alternativas y potencial de comercialización para los productos y especies de unidades productivas con escala mínima rentable del Estado de Puebla. México; 2014. http://www.sagarpa.gob.mx/ Delegaciones/puebla/Documents/Difusi\%C3\%B3n/ALTERNATIVAS_POTENCIAL_ COMERCIALIZACI\%C3\%93N-Puebla.pdf. Consultado 4 Feb, 2017.

6. Espejel G, Barrera R, Rodríguez M, Santiago V. Caracterización de los productores y dinámica de adopción de innovación en el municipio de Villa Victoria, Estado de México. Ra Ximhai 2015;11:17-34.

7. Pérez HP, Vilaboa AJ, Chalate MH, Candelaría MB, Díaz RP, López OS. Análisis descriptivo de los sistemas de producción con ovinos en el estado de Veracruz, México. Rev Cient Univ Zulia 2011;21(4):327-334.

8. Arteaga CJD. Estrategias para la recuperación de la ovinocultura en México. $10^{\circ}$ Encuentro Nacional Ganadero 2013. http://www.cnog.org.mx/_documentos/memoria62013.pdf. Consultado 14 Abr, 2017.

9. Mondragón-Ancelmo J, Domínguez-Vara I, Rebollar-Rebollar S, Bórquez-Gastélum J, Hernández-Martínez J. Margins of sheep meat marketing in Calpulhuac, state of Mexico. Trop Subtrop Agroecosystems 2012;15:105-116.

10. Mondragón-Ancelmo J, Hernández-Martínez J, Rebollar-Rebollar S, Salem AZM, Rojo-Rubio R, Domínguez-Vara IA, et al. Marketing of meat sheep with intensive finishing in southern state of Mexico. Trop Anim Health Prod 2014;46(8):1427-1433.

11. Salcedo S. Impactos diferenciados de las reformas sobre el agro mexicano: productos, regiones y agentes. Comisión Económica para América Latina y el Caribe (CEPAL). Santiago de Chile 1999. www.cepal.org/publicaciones/xml/7/4627/lcl1193p.pdf. Consultado 18 Ago, 2016. 
12. Gopinath M, Arnade C, Shane M, Roe L. Agricultural competitiveness: the case of U. S. and major EU countries. Agric Econ 1997;16(2):99-109.

13. Benzaquen J, Del Carpio LA, Zegarra LA, Valdivia CA. Un índice regional de competitividad para un país. Rev CEPAL 2010;102:69-86.

14. Vollrath T. A theoretical evaluation of alternative trade intensity measures of revealed comparative advantage. Weltwirtschaftliches Archiv 1991;127(2):265-279.

15. Osivweneta-Ogbe A, Olusegun-Okoruwa V, Jelili-Saka O. Competitiveness of Nigerian rice and maize production ecologies: a policy analysis approach. Trop Subtrop Agroecosystems 2011;14:493-500.

16. Monke EA, Pearson SR. The Policy Analysis Matrix for agricultural development. Ithaca, USA: Cornell University Press; 1989. doi:10.1080/03768359008439507.

17. Pearson S, Gotsch C, Bahri S. Applications of the Policy Analysis Matrix in Indonesian Agriculture. 2003. http://web.stanford.edu/group/FRI/indonesia/newregional/newbook.pdf. Consultado 18 Ago, 2016.

18. INEGI. Prontuario de información geográfica municipal de los Estados Unidos Mexicanos Naupan, Puebla, clave geoestadística 21100. 2009.

19. Scheaffer RL, Mendenhall W, Ott L. Elementos de muestreo. 6ta ed. Madrid, España: Thomson-Paraninfo S.A.; 2007.

20. Parra RI, Magaña MA, Duarte JH, Téllez IG. Caracterización técnica y rentabilidad de granjas ovinas con visión empresarial del Departamento del Tolima. Rev Colomb Cienc Anim 2014;7(1):64-72.

21. Salcedo BS. Competitividad de la agricultura en América Latina y el Caribe. Santiago, Chile: FAO; 2007. http://www.cepal.org/sites/default/files/courses/files/03_3_map_manual_fao.pdf. Consultado 16 Oct, 2016.

22. SNIIM. Sistema Nacional de Información de Integración de Mercados. Precios de mercado agrícola y pecuario. México. 2016. http://www.economiasniim.gob.mx/nuevo/. Consultado 16 Oct, 2016.

23. González-Garduño R, Blardony-Ricardez K, Ramos-Juárez J, Ramírez-Hernández B, Sosa R, Gaona-Ponce M. Rentabilidad de la producción de carne de ovinos Katahdin x Pelibuey con tres tipos de alimentación. Av Invest Agropecu 2013;17(1):135-148. 
24. Rebollar-Rebollar A, Hernández-Martínez J, Rebollar-Rebollar S, Guzmán-Soria E, García-Martínez A, González-Razo FJ. Competitividad y rentabilidad de bovinos en corral en el sur del Estado de México. Trop Subtrop Agroecosyst 2011;14:691-698.

25. Sosa MM, García MR, Omaña SJM, López DS, López LE. Rentabilidad de doce granjas porcícolas en la Región Noroeste de Guanajuato en 1995. Agrociencia 2000;34(1):107103.

26. Perdana T. Competitiveness and comparative advantage of beef cattle fattening in Bandung Regency. Badung: Research Institute Universitas Padjadjaran; 2003. http://web.stanford.edu/group/FRI/indonesia/research/beef.pdf. Consultado 26 Ago, 2016.

27. Babiker BI, Abdullah AJM, Al-Feel MA. Sudanese live sheep and mutton exports competitiveness. Saudi Soc Agric Sci 2011;10(1):25-32.

28. Góngora-Pérez RD, Góngora-González SF, Magaña-Magaña MA, Lara LE. Caracterización técnica y socioeconómica de la producción ovina en el estado de Yucatán, México. Agron Mesoam 2010;21(1):131-144.

29. Lustig NC, Székely M. México: evolución económica, pobreza y desigualdad. Washington D.C.; 1997. http://services.iadb.org/wmsfiles/products/Publications/ 816043.pdf. Consultado 22 Ene, 2016.

30. Rodríguez HR, Cadena IP, Morales GM, Jácome MS, Góngora GS, Bravo ME. Competitividad de las unidades de producción rural en Santo Domingo Teojomulco y San Jacinto Tlacotepec, Sierra sur, Oaxaca, México. ASyD 10: 2013;10(1):111-126.

31. Latruffe L. Competitiveness, productivity and efficiency in the agricultural and agrifood sectors. OECD Food, Agric Fish Pap 2010;30(30):1-63.

32. Carrera CB, Bustamante LTI. ¿Es la ganadería bovina de carne una actividad competitiva en México? Nóesis 2013;22(43):18-50.

33. Baldwin RE. Trade negotiations within the GATT/WTO framework: A survey of successes and failures. J Policy Model 2009;31(4):515-525.

34. Macedo R, Castellanos Y. Rentabilidad de un sistema intensivo de producción ovino en el trópico. Av Invest Agropecu 2004;8(May):1-9.

35. Barrera-Rodríguez AI, Jaramillo-Villanueva JL, Escobedo-Garrido JS, Herrera-Cabrera BE. Rentabilidad y competitividad de los sistemas producción de vainilla (Vanilla planifolia J.) en la región del Totonacapan, México. Agrociencia 2011;45(5):625-638. 